\title{
An Improved Assay and Tools for Measuring Mechanical Nociception in Drosophila Larvae
}

\author{
Roger Lopez-Bellido ${ }^{1}$, Michael J. Galko ${ }^{1,2,3}$ \\ ${ }^{1}$ Department of Genetics, The University of Texas MD Anderson Cancer Center ${ }^{2}$ Neuroscience Graduate Program, Graduate School of Biomedical \\ Sciences, The University of Texas MD Anderson Cancer Center ${ }^{3}$ Genetics and Epigenetics Graduate Program, Graduate School of Biomedical Sciences, \\ The University of Texas MD Anderson Cancer Center
}

\section{Corresponding Authors}

Roger Lopez-Bellido

Rlopez5@mdanderson.org

Michael J. Galko

mjgalko@mdanderson.org

\section{Citation}

Lopez-Bellido, R., Galko, M.J. An Improved Assay and Tools for Measuring Mechanical Nociception in Drosophila Larvae. J. Vis. Exp. (164), e61911, doi:10.3791/61911 (2020).

\section{Date Published}

October 29, 2020

DOI

$10.3791 / 61911$

URL

jove.com/video/61911

\section{Abstract}

Published assays for mechanical nociception in Drosophila have led to variable assessments of behavior. Here, we fabricated, for use with Drosophila larvae, customized metal nickel-titanium alloy (nitinol) filaments. These mechanical probes are similar to the von Frey filaments used in vertebrates to measure mechanical nociception. Here, we demonstrate how to make and calibrate these mechanical probes and how to generate a full behavioral dose-response from subthreshold (innocuous or non-noxious range) to suprathreshold (low to high noxious range) stimuli. To demonstrate the utility of the probes, we investigated tissue damageinduced hypersensitivity in Drosophila larvae. Mechanical allodynia (hypersensitivity to a normally innocuous mechanical stimulus) and hyperalgesia (exaggerated responsiveness to a noxious mechanical stimulus) have not yet been established in Drosophila larvae. Using mechanical probes that are normally innocuous or probes that typically elicit an aversive behavior, we found that Drosophila larvae develop mechanical hypersensitization (both allodynia and hyperalgesia) after tissue damage. Thus, the mechanical probes and assay that we illustrate here will likely be important tools to dissect the fundamental molecular/genetic mechanisms of mechanical hypersensitivity.

\section{Introduction}

Drosophila larvae exhibit a characteristic aversive rolling behavior when exposed to different noxious stimuli: thermal ${ }^{1}$, mechanical $^{2}$, and chemical ${ }^{3}$. This behavior is clearly distinct from normal locomotion. Here we describe an improved mechanical assay that can be used to assess mechanical nociception and mechanical sensitization.

In a recent study, we fabricated von Frey-like filaments using nitinol wires ${ }^{4}$. Probes exerting different forces and pressures were made by varying the lengths and diameters of the 
nitinol wires forming each probe. Mechanical probes were calibrated and the measured force values (in millinewton, $\mathrm{mN}$ ) were converted to pressure (kilopascal, kPa), based on the tip area of each probe ${ }^{4}$. Custom fabrication of mechanical probes allowed to us to generate subthreshold ( $\leq 200 \mathrm{kPa}$ ) to suprathreshold (225 kPa to $5318 \mathrm{kPa}$ ) pressures, which could, in principle, be beneficial for studying mechanical hypersensitivity. Using these improved mechanical von Freylike filaments, we showed that pressure ${ }^{4}$, as opposed to the previously examined force $2,5,6$ correlates more consistently with aversive behavioral responsiveness in Drosophila larvae. The improved mechanical assay described here also helped to identify a conserved Vascular Endothelial Growth Factor (VEGF)-related receptor tyrosine kinase signaling a pathway that regulates mechanical nociception in flies and rats ${ }^{4}$.

Mechanical allodynia and hyperalgesia, two modalities of hypersensitivity, are relatively understudied in Drosophila larvae, compared to the thermal (heat and cold) and chemical sensory modalities $3,7,8,9,10$. This is probably due to the lack of specific mechanical probes that span from innocuous stimuli to the high noxious range $2,5,6$. A normally innocuous stimulus that elicits the typical aversive rolling behavior after Drosophila larvae experience tissue damage da $^{3,7}$ is referred to as allodynia. An exaggerated rolling response to a typically noxious stimulus is known as hyperalgesia ${ }^{7}$. Noxious stimuli are defined as those that elicit tissue damage and can activate nociceptors ${ }^{11}$. Noxious stimuli delivered to Drosophila larvae damage either the barrier epidermis, the peripheral nociceptive sensory neurons ${ }^{3,4,7}$, or both.

In this article, we demonstrate how to custom fabricate and calibrate von Frey-like mechanical probes that are appropriate for Drosophila larvae. Further, we show how to use these probes to assay mechanical nociceptive responses in Drosophila larvae. Finally, we further demonstrate the utility of these probes by using them to demonstrate the presence of mechanical hypersensitivity, both allodynia and hyperalgesia, following tissue damage in Drosophila larvae (see Representative Results).

\section{Protocol}

\section{Mechanical probe construction}

1. Cut each nitinol filament (Figure 1B), perpendicular to its long axis, to the specified length (Figure $\mathbf{1} \mathbf{M}-\mathbf{N}$ ) using a small wire cutter (Figure 1C). The filaments come in three different pre-set diameters (Figure 1B).

NOTE: The lengths specified here are a guide to achieve the approximate pressures indicated, using a similar protocol for construction of the mount. Ultimately, regardless of the length of the filament cut, and the depth of the hole in the mount, the filaments must be measured/ calibrated on a balance to obtain the exact force/pressure value.

2. Examine the tip of the filament under a stereomicroscope to ensure no sharp or irregular edges remain as these could cause tissue damage to the skin of the larvae and interfere with calibration.

3. Manually smoothen the sharp edges of the mechanical probe using a sharpening stone until no sharp irregularities persist (Figure 1D).

4. Make a hole towards the end of a wooden popsicle stick (Figure 1E) using a hypodermic needle (see Table of Materials). Insert the needle at least halfway through the height of the popsicle stick (Figure 1E). This creates a chamber for insertion of the nitinol filament. 
5. Apply wood glue to a single nitinol filament (Figure 1F) and insert the glue-coated filament into the needle slot in a wooden popsicle stick (Figure 1G). Allow to dry for $\sim 5 \mathrm{~h}$.

6. Calibrate each mechanical probe by pressing it against a scale until the mechanical probe bends (Figure 1H-L). This is the point of maximum force that can be recorded in grams. Depending on the filament diameters (pre-set) and lengths (user-determined) a full range of forces and pressures can be generated.

7. Convert the mass recorded in step 1.6 to force in millinewton $(\mathrm{mN})$ using the formula $\mathrm{f}=\mathrm{ma}$ (Force is equal to mass multiplied by gravitational acceleration). f: force; m: mass; a: gravitational acceleration $\left(9.8 \mathrm{~m} / \mathrm{s}^{2}\right)$ (Figure $1 \mathrm{M})$.

8. Finally, convert the calculated force to pressure (force/ area) in kilopascal $(\mathrm{kPa})$ by dividing the measured force by the surface area of the filament tip (Figure 1M). To calculate the area, convert the diameter of the different nitinol filaments from inches (0.04", 0.06 ", and 0.08 ") to centimeters. Then, $\pi r^{2}$ (where, $r=$ the nitinol filament radius) determines the area (see Figure 1M). Preparing multiple probes using filaments of different diameters and lengths will generate a full set spanning the responsive range for Drosophila larvae (sample set shown in Figure $1 \mathrm{~N})$.

NOTE: Check each mechanical probe at least every 3-4 weeks. When the pressure deviates by more than $\pm 3 \%$ from the original measure, a new mechanical probe must be fabricated.

\section{Preparation of larvae}

1. Raise control strain $\left(w^{1118}\right)$ larval progeny or larvae containing the transgenes ppk-Gal4>UAS-mCD8-GFP (for visualizing damage to sensory neurons) on standard food in a $25{ }^{\circ} \mathrm{C}$ incubator. Typically, stocks are routinely maintained at $18{ }^{\circ} \mathrm{C}$ but both parents and larval offspring are reared at $25{ }^{\circ} \mathrm{C}$ on standard cornmeal food for experiments.

NOTE: Adult flies (five males and ten females, 1:2 ratio) are kept in the fly vials, to allow egg laying, for about 24 h. The time after egg laying (AEL) begins from when the adults are removed.

2. Collect the third instar larvae, after approximately $96 \mathrm{~h}$ of egg laying, by gently squirting tap water into the soft fly food containing the larvae. Wandering larvae that have left the food, or which have everted anterior or posterior spiracles, are too large/old for this assay. Second instar larvae ( less than $4 \mathrm{~mm}$ in length) are too small.

3. Pour out the contents of the soft fly food into a clean standard size Petri dish (100 mm x $15 \mathrm{~mm})$.

4. Using forceps, sort mid third instar, medium sized, larvae (see Figure 2A) from smaller (second instar and early third instar) or larger (late or wandering third instar) larvae. Gentle manipulation with forceps to avoid any tissue damage to the larvae is recommended.

NOTE: The transfer using forceps is based mostly on water tension and not by applying pressure to the larvae with the blades of the forceps. An alternative to the use of forceps for maneuvering larvae is soft paint brushes. With either tool, the user should practice transferring the animals, so as not to cause unintended tissue damage that could complicate behavioral measurements.

5. Transfer the mid third instar larvae, using forceps, into a small Petri dish $(30 \mathrm{~mm} \times 15 \mathrm{~mm})$ containing a small plug of fly food moistened with water at room temperature. Keep the larvae in the small Petri dish until 
the experiments are performed, but not longer than 20 $\min$.

NOTE: Generally, transferring 20-30 larvae to the food plug will give an adequate number for $20 \mathrm{~min}$ of behavioral assays.

\section{Mechanical nociception assay}

1. Place a mid-third instar larva (using forceps) onto a thin pad of black or dark vinyl under a bright field stereomicroscope. The dark color provides contrast that improves visualization of the larva. It is preferable to have a freely movable piece of dark vinyl because it allows the user to align the larva without touching or hurting it.

2. Put the optical fiber lights between the microscope objective lenses and the black or dark vinyl pad; this will allow adequate high contrast illumination for seeing the larva.

3. Discard larvae that do not exhibit normal locomotion following transfer to the pad. These can interfere with the normal nociceptive behavioral response. For normal locomotion, see Video 1.

4. Wipe away, using a paper towel, any excess water surrounding the larva that might cause the larva to float on the vinyl pad.

5. Orient the larva by moving the dark vinyl pad. The head/ mouth of the larva should point to the left if you are righthanded and vice versa if you are left-handed (Figure 2AB).

6. Apply the chosen mechanical probe, typically for $1-2 \mathrm{~s}$, onto the posterior dorsal side of the larva at approximately abdominal segment A8 (see Figure 2B), until the probe bends and elicits the previously measured amount of pressure (Figure $\mathbf{2 C}$ ). It is important that the probe presses against the dorsal surface of the larva and compresses the larvae into the underlying pad at the point of probe contact.

NOTE: At the point of contact between the tip of the nitinol filament and the dorsal cuticle-epidermis, probes lower than $2,300 \mathrm{kPa}$, mainly bend without penetrating the cuticle and underlying tissues. Such probes seldom affect larval mortality ${ }^{4}$. At higher pressures $(>5,000 \mathrm{kPa})$ the probes both bend and, occasionally, penetrate the cuticle and underlying tissues. Puncturing of the larvae impairs larval survival ${ }^{4}$ and, if observed, these larvae are typically discarded from behavioral analysis.

7. Record the behavioral response for each larva. A positive nociceptive response (Video 2 ) is indicated if the larva shows a complete roll of $360^{\circ}$ along the axis of its body within $3 \mathrm{~s}$. Other responses (attempting to turn, fast crawling, and wiggling) are considered negative for the purposes of this assay.

NOTE: Larvae stimulated with a subthreshold mechanical stimulus $(200 \mathrm{kPa})$ did not elicit the typical nociceptive or rolling response (Video 3 ). Some larvae did exhibit fast forward or light touch responses such as changes in the direction of movement.

8. Discard the larva and prepare the next one for assay, repeating steps 3.1 through 3.7 .

9. Repeat steps 3.1-3.7 until the desired number of larvae is reached (three to six sets of $n=10$ larvae were used here for each probe).

NOTE: When using lower pressure mechanical probes (174-462 kPa), the assay will take more time per larva. This is because the tip of longer filaments oscillates more, making it harder to poke the larva in the center of the A8 segment. Practice is necessary with these probes. 


\section{Confocal microscopy to assess neuronal morphology}

1. Place a larva (of genotype $p p k-G a / 4>U A S-m C D 8-G F P$ to label sensory neurons) previously stimulated with a nitinol filament into an etherization chamber inside a Coplin jar containing a $10 \mathrm{~mL}$ beaker carrying a cotton ball soaked with $\sim 1 \mathrm{~mL}$ of diethyl ether. Let the larva sit in the chamber for $\sim 5 \mathrm{~min}$.

NOTE: A detailed protocol for etherization is provided in a previous study published by our group ${ }^{12}$.

2. Rinse the larva gently from the etherization chamber into a small Petri dish.

3. Have ready one microscope slide, two small coverslips (22 x $22 \mathrm{~mm}$ ), and one long coverslip (22 x $54 \mathrm{~mm}$ ) (see Table of Materials).

4. Add small drops of ether:oil solution (1:5 ratio of ethyl ether to halocarbon oil solution, see Table of Materials) to both ends of the slide, then place the small coverslips on top of the small droplets. This arrangement creates a small space gap where the larva can fit.

NOTE: Press the small coverslips against the microscope slide until it is difficult to slip.

5. Add some drops of ether:oil solution on the middle of the microscope slide and then place the larva, using forceps, onto the center of the microscope slide (between the small coverslips). Make sure that the anteroposterior axis of the larva is parallel to the short side of the slide and that the dorsal side is facing up.

6. Cover the larvae with the long coverslip placed on top of the larva and the two smaller coverslips.

NOTE: Generously press the long coverslip until the larva is almost flat.
7. Image segment A8 of the larva using a confocal microscope (see Table of Materials) using laser wavelength 488 (GFP).

NOTE: Image the larva immediately because the anesthetization via ether will fade quickly $(\sim 5-10 \mathrm{~min})$ and the larva will wake up and move, which will complicate further imaging.

8. Capture Z-stack images at a resolution of $1024 \times 1024$ pixels using a 20x numerical aperture (NA) 0.7 dry objective lens at $1 \times$ zoom, step size of $1.5 \mu \mathrm{m}$.

\section{Quantitation of tissue damage}

1. Collect and convert the Z-series stack images, from section 4.8, into a single $\mathrm{Z}$ projection (a flattening of multiple images taken at different focal planes into a single composite image). This can be performed using commercially available software (e.g., Olympus Fluoview) or any equivalent open source platform, e.g., Fiji/lmage J. Save the single $Z$ projection in the TIFF format.

2. Open the image analysis program Fiji/lmageJ.

3. Click on File, from the menu bar, and select Open from the window that is displayed.

4. Select the stored single image projection, saved in the TIFF format, to be analyzed.

5. Click on Edit, from the menu bar, and select the Invert option from the window that is displayed.

6. Click on the Image, from the menu bar, then select Adjust, from the window that is displayed, and finally select the Brightness/Contrast option.

7. Select the Freehand Shape option from the tool bar to measure the area of the gap (if any). 
8. Click on Analyze, from the menu bar, and select the Measure option. This will display the area of the gap or wound.

\section{Representative Results}

We developed customized mechanical probes, using nitinol filaments (Figure 1A,N), to elicit mechanically-evoked behaviors and generated a full behavioral dose response curve using both innocuous and noxious mechanical probes of varying intensity (Figure 2D) demonstrating that these probes can be used to study baseline (in the absence of injury) mechanical nociception.

Our behavioral assay results determined that probes exerting pressures below $200 \mathrm{kPa}(\sim 1.57 \mathrm{mN})$ (Figure 1M), when applied to Drosophila larvae, do not provoke an aversive rolling response (Figure 2D and Video 3). As expected, these subthreshold or non-noxious mechanical probes (175 kPa or $200 \mathrm{kPa}$ ) did not elicit visible neuronal tissue damage (Figure 2E). Because they do not induce damage, such probes could be useful to assess mechanical allodynia (hypersensitivity to a normally non-noxious mechanical stimuli). Conversely, suprathreshold or noxious probes (from $462 \mathrm{kPa}$ to $5,116 \mathrm{kPa}$ ), elicited an augmented behavioral response (Figure 2D) in a dose dependent manner-with the higher pressures eliciting stronger behavioral responses. As anticipated, suprathreshold mechanical pressure also induced dose-dependent tissue damage to the peripheral sensory neurons themselves (Figure 2E). The measured area of tissue damage (in $\mu \mathrm{m}^{2} \pm$ standard deviation) taken from four larvae for each group were: 2,051.03 \pm 703.81 (462 kPa), 5,102.29 \pm 1,004.67 (2,283 kPa), and 12,238.83 $\pm 3,724.11(5,116 \mathrm{kPa})$. Thus, pressures greater than or equal to $462 \mathrm{kPa}(\sim 63 \mathrm{mN})$, which evoke an aversive rolling response (in $25 \%$ or more of the larvae) and cause visible neuronal tissue damage (Figure 2E), could be appropriate to study mechanical hyperalgesia (hypersensitivity to normally noxious mechanical stimuli). Nociceptive mechanical probes $(\geq 462 \mathrm{kPa}$ ) always induce tissue damage $(\mathrm{n}=10$, evaluated qualitatively) but do not always provoke an aversive rolling response.

To evaluate mechanical hypersensitivity (allodynia and hyperalgesia), we used a well-established Drosophila larval model of nociceptive sensitization that uses ultraviolet light (UV) irradiation to induce tissue damage ${ }^{7,12}$. This assay has helped to dissect the genetic and cellular mechanisms of thermal nociceptive hypersensitivity $8,9,10,13,14,15$. To determine whether UV treatment causes mechanical allodynia, mid third-instar control $\left(w^{1118}\right)$ larvae were mockirradiated or UV-irradiated $\left(15-20 \mathrm{~mJ} / \mathrm{cm}^{2}\right)$ (Figure 3A). Then, the larvae were tested behaviorally at $2 \mathrm{~h}, 4 \mathrm{~h}, 8 \mathrm{~h}$, $16 \mathrm{~h}$, and $24 \mathrm{~h}$ post-treatment with a normally subthreshold mechanical probe (200 kPa, $1.57 \mathrm{mN}$ ). Approximately $20 \%$ of larvae responded as early as $2 \mathrm{~h}$ after UV treatment while $50 \%$ responded at $4 \mathrm{~h}$, compared to $6.6 \%$ and $8.3 \%$ mock UVirradiated animals, respectively (Figure 3B). This indicates that UV-induced tissue damage causes mechanical allodynia at $4 \mathrm{~h}$ post-irradiation. At later time points $(8 \mathrm{~h}, 16 \mathrm{~h}$, and 24 h) the behavioral response of the UV-treated larvae was in the range of $16 \%-20 \%$ responders (average mean of $n=3-6$ sets of 10 larvae each), slightly increased (but not statistically significant) compared to the mock-irradiated control group (in the range of $3 \%-6 \%$ of responders, average mean of $n=3-$ 6 sets of 10 larvae each) (Figure 3B).

To investigate mechanical hyperalgesia, a suprathreshold pressure $(462 \mathrm{kPa}, 3.63 \mathrm{mN})$, that normally induces an aversive rolling response in $\sim 20 \%$ of larvae (Figure 2D) and causes neuronal tissue damage (Figure 2E), was used. 
We applied the $462 \mathrm{kPa}$ probe onto the dorsal side of larvae with or without UV-induced tissue damage (Figure 3A). We found that larvae probed at $4 \mathrm{~h}, 8 \mathrm{~h}$, and $16 \mathrm{~h}$ following UV treatment showed a significant increase in the aversive rolling response, with $4 \mathrm{~h}$ being the peak of the behavioral hypersensitivity ( $60 \%$ responsive); mock UVirradiated animals showed an $\sim 27 \%$ of aversive response (Figure 3C). Similar to mechanical allodynia, the behavioral response at $8 \mathrm{~h}, 16 \mathrm{~h}$, and $24 \mathrm{~h}$ of UV-treated animals (in the range of $36 \%-42 \%$ ) was statistically indistinguishable from the non-treated larvae (in the range of $20 \%-26 \%$ ). Larvae at the late third instar stage did show a slight decrease of the baseline behavioral response when compared with the middle third instar stage. We hypothesize this could be either by the increased size of the larvae (Figure 2A) or the increased thickness of the cuticle covering the body. This fact could explain why at a later stage of development the UV treatment does not induce greater mechanical sensitization, as observed $4 \mathrm{~h}$ post UV treatment.

Taken together, our results indicate that Drosophila larvae develop both mechanical allodynia and mechanical hyperalgesia following UV-induced tissue damage. The peak time of mechanical allodynia and hyperalgesia is the same, 4 h after UV treatment; however, mechanical hyperalgesia has a more pronounced temporal tail as it returns to baseline more slowly compared to mechanical allodynia. 
A

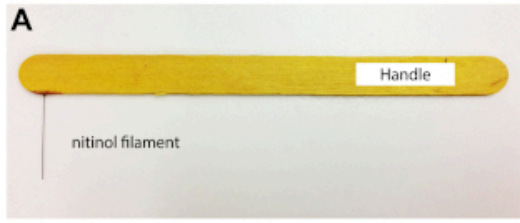

D

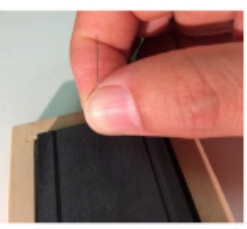

H

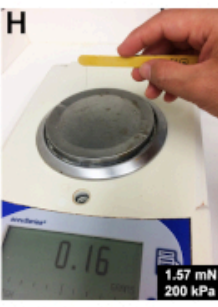

M

E

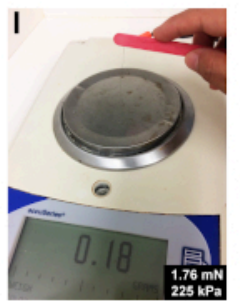

J
B
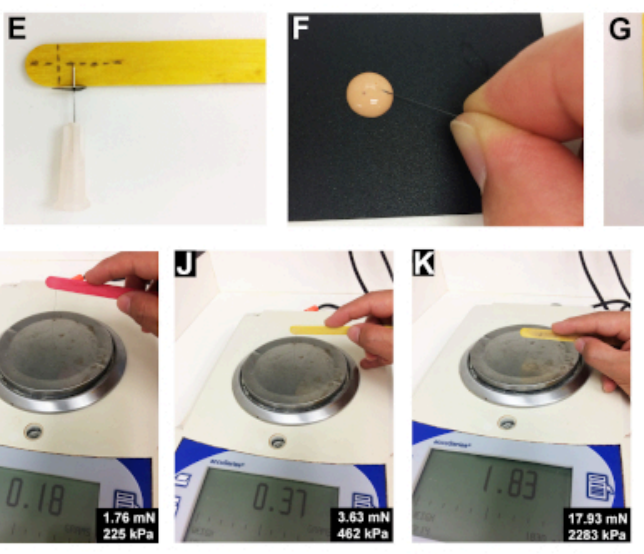

$\mathrm{L}$
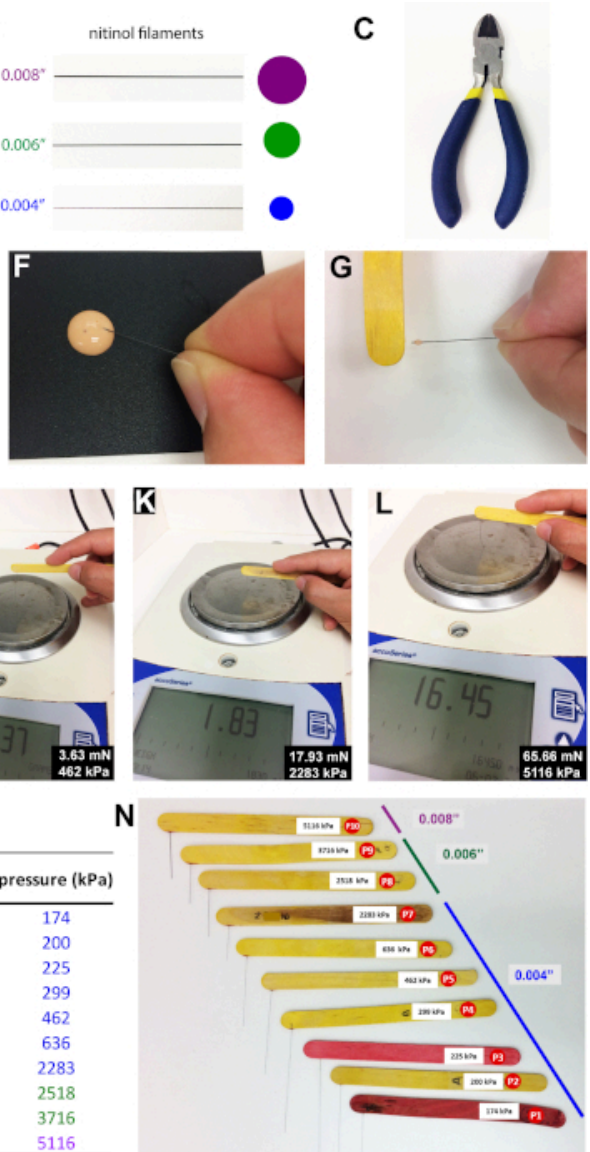

Figure 1: Development of a Von Frey-like tool to evaluate mechanical nociception in Drosophila larvae. (A) Picture of a mechanical probe used to study mechanical nociception in Drosophila larvae. (B) Nitinol filaments and their relative diameters are shown to relative scale. (C) Picture of the diagonal wire cutter used to cut the nitinol filaments. (D) Smoothing the sharp edges of the cut nitinol filament with a sharpening stone. (E) Hypodermic needle used to make a hole into the wooden popsicle stick handle of the probe. The tip of the needle needs to reach at least half the height of the handle stick for secure filament insertion. (F-G) Attachment of the nitinol filament by gluing into a wooden popsicle stick handle with insertion hole. (H-L) Calibration of mechanical probes by pressing them against a scale. (M) Values of force (in $\mathrm{mN}$ ) and pressure (in $\mathrm{kPa})$ generated by different mechanical probes. The length of each nitinol filament used to construct the probes (P1-P10; P: probe) is detailed in centimeters $(\mathrm{cm})$. (N) A picture of a complete set of mechanical probes, ranging from $174 \mathrm{kPa}$ to 5,116 $\mathrm{kPa}$. Please click here to view a larger version of this figure. 


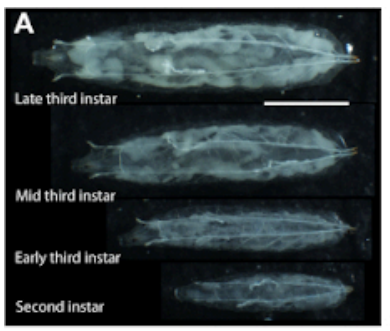

B

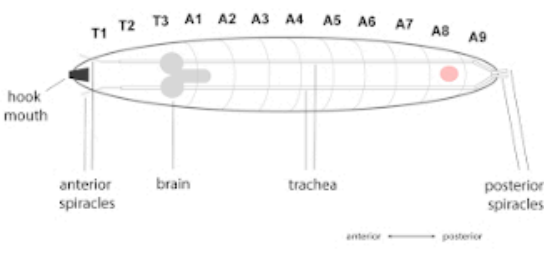

C

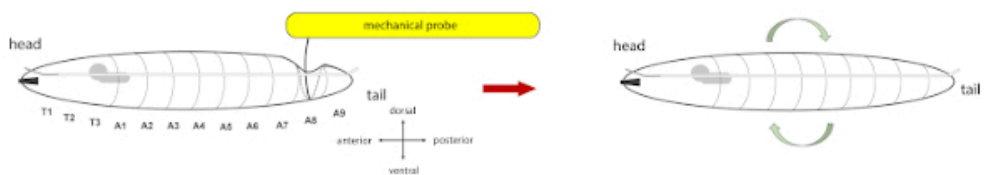

D

E
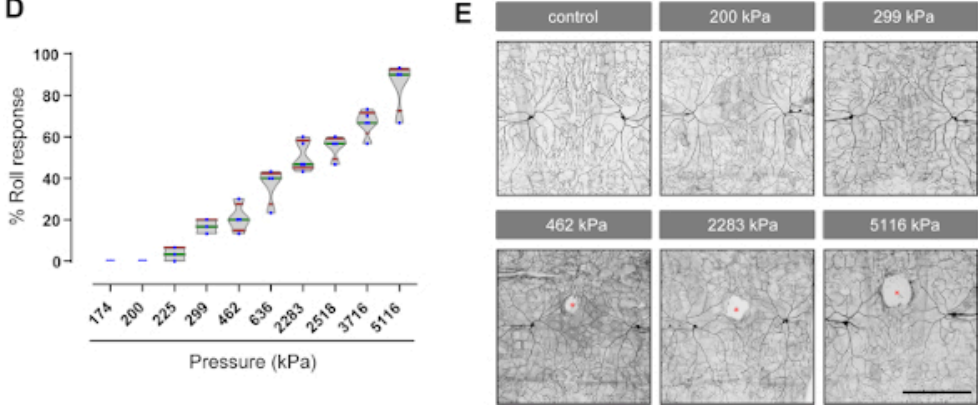

Figure 2: Mechanical nociception assay: Von Frey-like filaments generate a dose-response curve of aversive rolling behavior and cause tissue damage to sensory neurons. (A) Pictures of the different stages (second and third instar) of Drosophila larvae. Scale bar: $2 \mathrm{~mm}$. (B) Cartoon of the dorsal view of the third instar Drosophila larvae. The red dot indicates the abdominal segment where the mechanical probe is applied. T: thoracic segment; A: abdominal segment. Other anatomical landmarks are labeled. (C) Cartoon of the assay: A mechanical probe is applied to the dorsal side of the larva until it bends against the surface below and is then held for $2 \mathrm{~s}$. If the pressure is sufficiently high, this elicits an aversive rolling response upon release. (D) Behavioral dose response; each blue dot represents the percent of larvae that responded, with aversive rolling, to the mechanical stimulation within a set of 10 animals. Violin plot of the percent of aversive rolling behavior induced by different mechanical probes. kPa: kilopascals. Box plots represent median (green), whiskers (red) represent the 10th and 90th percentiles. (E) Tissue damage: Third instar larvae (of genotype ppk-Gal4>UAS-mCD8-GFP to label nociceptive sensory neurons) were probed at dorsal segment A8 with the indicated pressures. Fluorescently labeled paired ddaC class IV sensory neurons (across the dorsal midline) were then examined (see sections 4 and 5 ). White areas (red asterisks) represent gaps or tissue damage. Scale bar: $100 \mu \mathrm{m}$. In panel B, the larva is shown in the dorsal view, while in $\mathrm{C}$ it is the lateral view. Mechanical probes pressed against the dorsal cuticle-epidermis side of the larva produce a depression like-pocket at the point of contact of the tip of the probe and the surrounding areas. The solid black line curved 
toward the ventral side is the top of the pocket, while the dashed gray lateral line represents the lateral side and the bottom of the pocket. Please click here to view a larger version of this figure. 
A
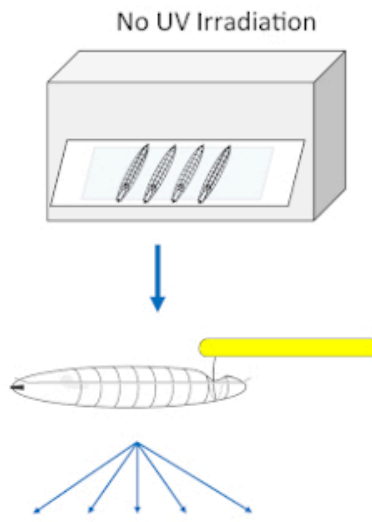

$\frac{2 \mathrm{~h} \quad 4 \mathrm{~h} \quad 8 \mathrm{~h} \quad 16 \mathrm{~h} \quad 24 \mathrm{~h}}{\text { After mock treatment }}$

B

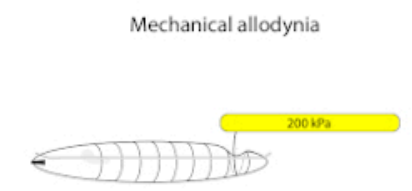

C

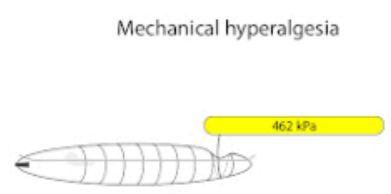

UV Irradiation
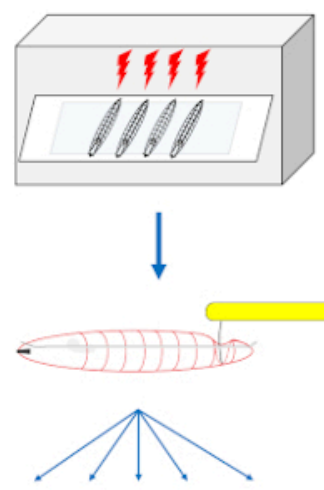

$\frac{2 \mathrm{~h} 4 \mathrm{~h} \quad 8 \mathrm{~h} 16 \mathrm{~h} \quad 24 \mathrm{~h}}{\text { After UV irradiation }}$
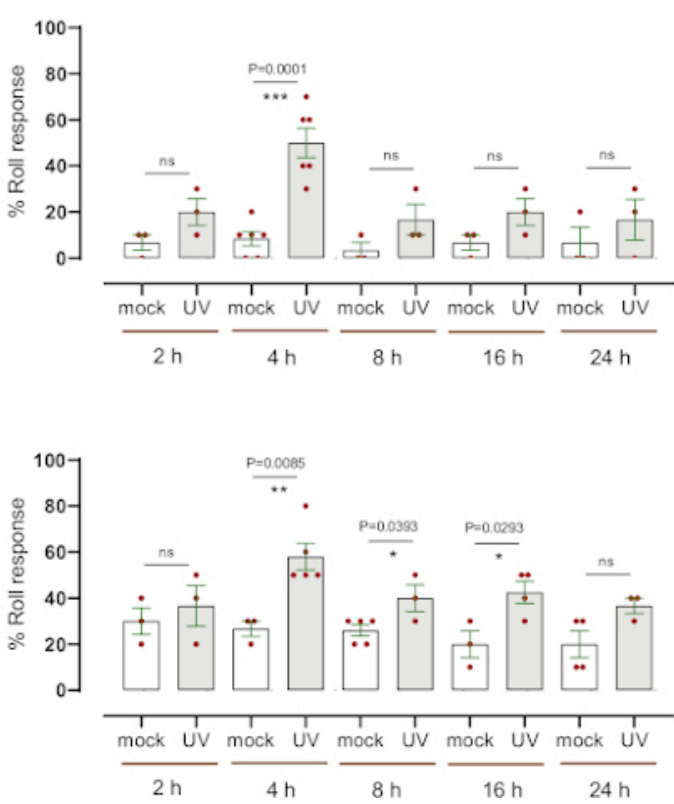

Figure 3: Mechanical hypersensitivity after UV damage. (A) Schematic of the experimental design to test sensitization. Mid third instar were mock treated (non-UV) or UV irradiated. The mechanical nociception assay was then performed at different time points ( $2 \mathrm{~h}, 4 \mathrm{~h}, 8 \mathrm{~h}, 16 \mathrm{~h}$, and $24 \mathrm{~h}$ ) following mock treatment or irradiation. (B) Mechanical allodynia: The percentage of larvae exhibiting aversive rolling after probing with a normally subthreshold or non-noxious mechanical stimulus $(200 \mathrm{kPa}, 1.57 \mathrm{mN})$ at the indicated time points after mock-treatment or UV irradiation. (C) Mechanical hyperalgesia: The percentage of larvae exhibiting aversive rolling after probing with a normally suprathreshold or noxious mechanical stimulus (462 kPa, $3.63 \mathrm{mN}$ ) at the indicated time points after mock-treatment or UV irradiation. Error bars indicate mean $+/-$ SEM. Two-tailed unpaired $t$-test was used for statistical analysis: ${ }^{*} p<0.05$, ${ }^{* *} p<0.01$; ns: not significant. Each red dot, in 
panels $B$ and $C$, represents the mean proportion of 10 larvae, $n=3-6$ sets per timepoint/condition. Please click here to view a larger version of this figure.

Video 1: Normal locomotion of Drosophila larvae. Please click here to download this video.

\section{Video 2: Noxious mechanical stimulation of Drosophila larvae. Please click here to download this video.}

\section{Video 3: Subthreshold mechanical stimulation of} Drosophila larvae. Please click here to download this video.

\section{Discussion}

We modified an established mechanical assay ${ }^{1,2,16}$ using customized mechanical probes fabricated from nitinol filaments. This metal alloy allows us to use smaller diameter filaments that are appropriate to the size of the Drosophila larvae. Fishing line-based monofilaments have dominated the field of fly mechanical nociception to date $2,5,6,16$. Our nitinol filaments maintain their shape and measured pressure for approximately $\sim 3-5$ months (in our experience). By varying the length and diameter of the nitinol filaments, the user can generate a wide range of pressures spanning from subthreshold to a nearly complete rolling response. In particular, making subthreshold probes is simpler with the smaller diameter nitinol filaments. Using these probes, we found that pressure, rather than force, elicits more consistent nocifensive behavioral responses ${ }^{4}$. We demonstrate here, using a well-established UV-induced nociceptive sensitization model $^{7,10,13}$, that these filaments are also a useful tool for studying mechanical hypersensitivity—allodynia and hyperalgesia.

Previous studies using mechanical probes fabricated from fishing line have led to a certain variability in behavioral responsiveness $2,6,16,17$. Several factors may account for this. First, because pressure is the important variable, the buffing of the filament tip so that it is rounded and does not have any sharp edges is critical. Second, reporting pressure values rather than only force is important for the reproducibility of the experiments, because different mechanical probes that generate similar forces can elicit disparate pressures ${ }^{4}$. Third, it is critical to apply only one mechanical stimulation per larva using noxious probes, because such probes produce a dose-dependent tissue damage at the epidermal ${ }^{4}$ and sensory neuronal levels (Figure 2E). A second or subsequent noxious mechanical stimulus, after tissue damage has been induced, could conceivably impair the function of the affected peripheral sensory neurons and elicit an altered behavioral response. In another study, larvae stimulated twice with noxious mechanical probes mostly displayed an enhanced behavioral response ${ }^{5}$, suggesting development of an acute mechanical sensitization (hyperalgesia), which might result from the tissue damage provoked by the first noxious mechanical stimulus. Conversely, other authors ${ }^{6}$ reported a mixed (increased or decreased) behavioral response, indicating that the altered behavioral response could be due to damage/dysfunction of the neuronal tissue. Stimulating each larva only once eliminates possible variance in behavioral responses resulting either from sensitization or tissue damage. Fourth, we mechanically stimulated segment A8, which is more posterior than previous studies (preferred areas A3-A4) $2,5,16$. Probes between $\sim 3,900 \mathrm{kPa}$ and 5,300 $\mathrm{kPa}$ applied to either segment A2 or A8 did not show any behavioral differences ${ }^{4}$. In addition, A8, compared to $\mathrm{A} 2-\mathrm{A} 4$, is easier to stimulate with mechanical probes that generate lower pressures $(<300 \mathrm{kPa})$ because the larva is thinner in this region and thus more easily compressed. Other 
studies showed that noxious mechanical stimulation of the posterior end of the larva (delivered by a rigid insect pin, held with forceps) mostly evoked forward locomotion, rather than an aversive or rolling response ${ }^{18}$. This different behavioral response could be due to differences in the properties of the used materials (bendable nitinol filament vs incompressible insect pin) or to different pressures delivered to the larvae (the pressure value of the insect pin was not reported).

The development of a mechanical nociception assay for Drosophila larvae has enabled the field to discover that different mechanical sensory ion channels and neural circuits mediate mechanical nociception $5,6,16,17$. However, the study of the mechanical hypersensitivity (allodynia and hyperalgesia) has lagged, compared to sensitization of the other sensory modalities-heat ${ }^{7,8,10,13,14}$, cold $^{9}$, and chemical $^{3}$. This lag may be due in part to the absence of suitable mechanical probes that can generate a full response range spanning subthreshold to suprathreshold pressures. Of particular importance, especially for assessing mechanical allodynia, are subthreshold probes that do not elicit an aversive rolling response from uninjured larvae. The significance of our improved mechanical probes is that they can be fabricated to span innocuous stimuli (subthreshold $\sim 174 \mathrm{kPa}-200 \mathrm{kPa}$ ) or the low to high noxious range (suprathreshold $\sim 225 \mathrm{kPa}$ to $\sim 5,116 \mathrm{kPa}$ ). Here, we demonstrate using the nitinol von Frey-like filaments that Drosophila larvae develop both mechanical allodynia and mechanical hyperalgesia after UV irradiation. The mechanical sensitization shows some differences when compared to thermal sensitization. Both the onset and the peak of mechanical sensitization is earlier ( 4 h) compared to thermal (heat) sensitization ( $8 \mathrm{~h}$ for hyperalgesia and $\sim 24 \mathrm{~h}$ for allodynia) ${ }^{7}$. In addition, the mechanical allodynia and hyperalgesia are concomitant (both peak at $\sim 4 \mathrm{~h}$ ). Furthermore, while heat sensitization (allodynia and hyperalgesia) resolves completely at later time points ${ }^{7}$, mechanical hypersensitivity exhibited a long tail that remained slightly above baseline. Cold sensitization in Drosophila involves a switch in cold-evoked behaviors ${ }^{9}$ and the emergence of new cold-evoked behaviorsa phenomenon that is not observed with mechanical stimulation. These differences in onset, duration, and observed behaviors suggest that each sensory modality may be controlled by different signaling pathways. Combining the sensitization assay described here with the powerful genetic tools available in Drosophila should allow a precise genetic dissection of the mechanical hypersensitivity (allodynia and hyperalgesia) observed.

\section{Disclosures}

The authors have nothing to disclose.

\section{Acknowledgments}

We thank Thomas Wang for developing the prototype von Frey filaments, Patrick J. Huang for improving the mechanical probe assay, the Bloomington Drosophila Stock Center for the control $\left(w^{1118}\right)$ and ppk-Gal4>UAS-mCD8-GFP fly stocks, and Galko lab members for critically reading the manuscript. This work was supported by R21NS087360 and R35GM126929 to MJG.

\section{References}

1. Tracey, W. D., Jr., Wilson, R. I., Laurent, G., Benzer, S. Painless, a Drosophila gene essential for nociception. Cell. 113 (2), 261-273 (2003).

2. Zhong, L., Hwang, R. Y., Tracey, W. D. Pickpocket is a $\mathrm{DEG} / \mathrm{ENaC}$ protein required for mechanical nociception 
in Drosophila larvae. Current Biology. 20 (5), 429-434 (2010).

3. Lopez-Bellido, R., Himmel, N. J., Gutstein, H. B., Cox, D. N., Galko, M. J. An assay for chemical nociception in Drosophila larvae. Philosophical Transactions of the Royal Society of London. Series B: Biological Sciences. 374 (1785), 20190282 (2019).

4. Lopez-Bellido, R. et al. Growth factor signaling regulates mechanical nociception in flies and vertebrates. Journal of Neuroscience. 39 (30), 6012-6030 (2019).

5. Hu, C. et al. Sensory integration and neuromodulatory feedback facilitate Drosophila mechanonociceptive behavior. Nature Neuroscience. 20 (8), 1085-1095 (2017).

6. Kim, S. E., Coste, B., Chadha, A., Cook, B., Patapoutian, A. The role of Drosophila Piezo in mechanical nociception. Nature. 483 (7388), 209-212 (2012).

7. Babcock, D. T., Landry, C., Galko, M. J. Cytokine signaling mediates UV-induced nociceptive sensitization in Drosophila larvae. Current Biology. 19 (10), 799-806 (2009).

8. Babcock, D. T. et al. Hedgehog signaling regulates nociceptive sensitization. Current Biology. 21 (18), 1525-1533 (2011).

9. Turner, H. N., Patel, A. A., Cox, D. N., Galko, M. J. Injuryinduced cold sensitization in Drosophila larvae involves behavioral shifts that require the TRP channel Brv1. PloS One. 13 (12), e0209577 (2018).

10. Im, S. H. et al. Tachykinin acts upstream of autocrine Hedgehog signaling during nociceptive sensitization in Drosophila. eLife. 4, e10735 (2015).
11. Cervero, F., Merskey, H. What is a noxious stimulus? Pain Forum. 5 (3), 157-161 (1996).

12. Chattopadhyay, A., Gilstrap, A. V., Galko, M. J. Local and global methods of assessing thermal nociception in Drosophila larvae. Journal of Visualized Experiments: JoVE. (63), e3837 (2012).

13. Follansbee, T. L. et al. Drosophila nociceptive sensitization requires BMP signaling via the canonical SMAD pathway. Journal of Neuroscience. 37 (35), 8524-8533 (2017).

14. Im, S. H., Patel, A. A., Cox, D. N., Galko, M. J. Drosophila insulin receptor regulates the persistence of injury-induced nociceptive sensitization. Disease Models \& Mechanisms. 11 (5) (2018).

15. Jo, J. et al. Drosophila caspase activity is required independently of apoptosis to produce active TNF/Eiger during nociceptive sensitization. Cell Death \& Disease. 8 (5), e2786 (2017).

16. Hwang, R. Y. et al. Nociceptive neurons protect Drosophila larvae from parasitoid wasps. Current Biology. 17 (24), 2105-2116 (2007).

17. Guo, Y., Wang, Y., Wang, Q., Wang, Z. The role of PPK26 in Drosophila larval mechanical nociception. Cell Reports. 9 (4), 1183-1190 (2014).

18. Takagi, S. et al. Divergent connectivity of homologous command-like neurons mediates segment-specific touch responses in Drosophila. Neuron. 96 (6), 1373-1387 e1376 (2017). 